Die Koeducation von männlichen und weiblichen Gefangenen wird nur im Bundesentwurf insoweit geregelt, als die gemeinsame Schul- und Berufsausbildung für zulässig erklärt wird ( $\$ 19$ Abs. 3 S. 2) - angesichts der Probleme gerade der männlichen jungen Gefangenen im Umgang mit dem weiblichen Geschlecht eine mehr als sinnvolle Maßnahme.

Weiterhin fordert der Entwurf des BJM verbindlich die Einzelunterbringung der Gefangenen (§ 19 Abs. 4 S. 1), während im baden-württembergischen Entwurf insoweit nur eine Soll-Vorschrift formuliert wird ( $§ 20$ Abs. 1); dies gilt auch für den bayerischen Entwurf (Art. 138 Abs. 1, Art. 20 Abs. 1).

Für zukünftige Jugendstrafanstalten - ab 2010 fordert der BJM-Entwurf eine Anstaltsgröße von höchstens 240 Haftplätzen, wobei die Wohngruppen auf max. 8 Personen begrenzt werden ( $\$ 40$ Abs. 3). Bis zum 1.1.2008 müssen nach diesem Entwurf die Bildungs- und Ausbildungsstätten so ausgebaut sein, dass für mindestens $2 / 3$ der Haftplätze Plätze für allgemeine und berufliche Bildung in Schulen und berufliche Ausbildungsstätten sowie für arbeitstherapeutische Maßnahmen zur Verfügung stehen (§ 40 Abs. 7). Für Neueinstellungen von Vollzugsbediensteten im Jugendstrafvollzug wird eine zusätzliche pädagogische Ausbildung von mindestens 6 Monaten verlangt (§ $41 \mathrm{Abs}$. 3).

Dies waren alles Positionen, die von den Ländern aus finanziellen Gründen abgelehnt wurden. Entsprechende Vorgaben finden sich dementsprechend nicht in den beiden Länderentwürfen. Nur hinsichtlich der pädagogischen
Ausbildung ist sowohl im baden-württembergischen Entwurf ( $\$ 42$ Abs. 3) als auch im bayerischen Entwurf (Art. 155) eine Soll-Bestimmung aufgenommen.

\section{6. Überprüfungs- und Nachbesserungs- pflicht}

Die kriminologische Forschung zur Überprüfung des Jugendstrafvollzugs hinsichtlich seiner Auswirkungen auf die Gefangenen wird in allen 3 Entwürfen vorgeschrieben ( $\$ 39$ BJM; $§ 40$ BadenWürttemberg; Art. 188, 120 Bayern). Abgesehen von der praktischen Umsetzung, d. h. der Einrichtung eines kriminologischen Dienstes, wird kritisch zu beobachten sein, wie mit solchen einheitlichen oder divergierenden Forschungsergebnissen umgegangen wird, d. h. ob hieraus Konsequenzen für Gesetzesänderungen gezogen werden. Im hessischen Jugendvollzug sollen zukünftig systematische Rückfalluntersuchungen eingeführt werden (s. Einheitliche Vollzugskonzeption im hessischen Jugendvollzug, Mai 2004, Ziff. 8).

\section{Neues Rechtsmittelsystem}

Das vom Bundesverfassungsgericht geforderte neue Rechtsmittelsystem wird sowohl im Bundesentwurf (§ 35) als auch im baden-württembergischen Entwurf ( $\$ 36)$ in Anlehnung an die Regelung im Strafvollzugsgesetz ( $\$ \S 108$ ff.) eingeführt. Allerdings entscheidet nach dem BJM-Entwurf die Jugendkammer - in der Regel durch ein Mitglied der Jugendkammer - über den Antrag auf gerichtliche Entscheidung ( $\$ 35$ Abs. 3,4$)$, nach dem baden- württembergischen Entwurf der Jugendrichter als Vollstreckungsleiter (§ 36 Abs. 3). Im bayerischen Entwurf ist eine Regelung zum Rechtsmittelsystem bislang vergessen worden mit Ausnahme des Beschwerderechts (Art 114). Bemerkenswert ist, dass § 74 JGG sowohl im BJM-Entwurf (§ 35 Abs. 5) als auch im baden-württembergischen Entwurf (§ 36 Abs. 4) für entsprechend anwendbar erklärt wird, d. h. von den Kosten des Rechtsmittels kann der Gefangene befreit werden.

\section{Zusammenfassende Bewertung}

Zusammenfassend ist festzuhalten, dass der Entwurf des BJM am ehesten den Anforderungen des Bundesverfassungsgerichts entspricht, weniger der baden-württembergische Entwurf und am wenigstens der bayerische Entwurf. Angesichts der festgestellten Unterschiede erscheint die Formulierung des baden-württembergischen Justizministers, dass Bayern und Baden-Württemberg einen „in wesentlichen Teilen aufeinander abgestimmten Entwurf eines Jugendstrafvollzugsgesetzes" vorlegen, schwer nachvollziehbar. Die weitere Formulierung, dass damit „sämtliche Vorgaben des Bundesverfassungsgerichts voll umgänglich erfüllt" werden, ist eine politische, sachlich nicht begründete Behauptung (s. Pressemitteilung des baden-württembergischen Justizministeriums vom 7.6.2006).

Der Verfasser ist Leiter der Forschungsstelle für Jugendstrafrecht und Kriminalprävention an der Universität Kiel und Mitherausgeber dieser Zeitschrift.

\title{
Optimale Förderung oder was sollte Jugendstrafvollzug leisten?
}

\section{Joachim Walter}

\section{Problemstellung}

Das Urteil des Bundesverfassungsgerichts vom 31.05.2006 (ZJJ 2006: 193) verpflichtet den Gesetzgeber, bis spätestens Ende 2007 ein Jugendstrafvollzugsgesetz zu erlassen. Gleichwohl sollen im Folgenden nicht konkret formulierte Vorschläge für die Vorschriften eines künftigen Jugendstrafvollzugsgesetzes vorgelegt werden (hierzu Walter 2003: 397 und ZfJ 2004, 397; DVIJ 2004: 209). Meine Thesen setzen vielmehr einen geeigneten rechtlichen Rahmen bereits voraus, wie ihn - trotz mancher Einschränkungen - beispielsweise der vom Bundesministerium der Justiz am 7. 6. 2006 der Öffentlichkeit vorgestellte Entwurf eines Jugendstrafvollzugsgesetzes durchaus darstellen könnte. Von den Essentialia, wie sie schon § 91 JGG oder der erwähnte Entwurf in den Mittelpunkt stellen - Schule, Arbeit, Berufsausbildung, Sport - wird, weil eigentlich selbstverständlich, weniger die Rede sein. Als Antwort auf die immer wieder geführte Klage über die ungünstigen tatsächlichen Rahmenbedingungen, unter denen der Jugendstrafvollzug heute zu arbeiten hat, soll vielmehr einmal versucht werden, eine - natürlich keineswegs abschließende - Positivliste der Bedingungen aufzustellen, die eine optimale Förderung Jugendlicher im Strafvollzug ermöglichen würden. Dabei geht es zwar auch um Ziele und den formellen Rahmen, mehr aber noch um dessen inhaltliche Ausgestaltung. Kurzum, es geht um das Setting und das Klima in der Jugendstraf- anstalt; Verhältnisse also, die sich nicht nur und vielleicht nicht einmal in erster Linie aus Rechtsvorschriften ergeben, sondern die die Praxis gestaltet.

Ich möchte damit zunächst zahlreiche positive Ansätze bekannt machen, unterstützen und zur Diskussion stellen, die in verschiedenen Jugendanstalten schon heute existieren, allerdings nur teilweise in der Fachliteratur beschrieben sind. Es wird jedoch nicht nur gefragt, was ohne weiteres getan werden könnte bzw. heute schon erreicht ist oder was wir uns (noch) leisten können. Vielmehr versuche ich darüber hinaus Antworten auf die Frage zu finden, welche Bedingungen in einem Jugendstrafvollzug herrschen müssten, um eine 
bestmögliche Förderung seiner Insassen während der Haft zu gewährleisten. Diese werden keineswegs nur von den rechtlichen Regelungen und den verfügbaren Ressourcen determiniert; mindestens ebenso wichtig ist das Klima in der Anstalt und die Art und Weise, wie der Jugendstrafvollzug tatsächlich „gelebt" wird. Dabei werden die Rahmenbedingungen von verschiedenen Standpunkten aus und damit in verschiedener Perspektive beschrieben: Derjenigen des Gefangenen, derjenigen der Mitarbeiter, mit Blick auf die Beziehung zwischen Mitarbeitern und Gefangenen und schließlich mit Blick auf die Lernumwelt, die die Anstalt darstellt und die sie durch ihre Organisationsstruktur beeinflusst. Solche Sicht aus unterschiedlicher Perspektive bringt allerdings mit sich, dass manche Themen wiederholt, wenn auch von verschiedenen Seiten, beleuchtet werden.

Die Darstellung der Rahmenbedingungen eines auf bestmögliche Förderung der jungen Menschen in ihrer Entwicklung angelegten Jugendstrafvollzugs zeichnet also in mancher Hinsicht ein Idealbild. Aus heuristischen Gründen und um mancher Idee nicht schon vor der Diskussion die Flügel zu stutzen, bleibt der aktuelle gesamtgesellschaftliche Hintergrund, was manchen „Realisten" stören mag, zunächst ausgeblendet. Diesen könnte man augenblicklich mit Schlagwörtern wie knappe Haushaltsmittel, steigende Kriminalitätsangst, zunehmende Punitivität in Politik und Rechtsprechung, aber auch wachsender Anteil der alten Menschen in der Gesellschaft oder Integrationsprobleme junger Migranten beschreiben. Was die Kosten angeht, um nur auf einen besonders wichtigen dieser Gesichtspunkte einzugehen, so werden die allermeisten Vorschläge keineswegs mit erhöhten Ausgaben verbunden sein. Vielmehr handelt es sich ganz überwiegend um eine differente Akzentsetzung, teilweise auch um ein bewusstes Umsteuern. Auch der Zeit- und Personalaufwand dürfte sich vom heutigen nicht erheblich unterscheiden, zumal viele Vorschläge bereits in der einen oder anderen Anstalt verwirklicht sind - freilich nirgendwo alle oder auch nur die Mehrzahl davon. Was allerdings nicht erspart werden kann, ist die Neu- und Umorientierung mancher Arbeitsabläufe sowie die Analyse bestehender Strukturen auf ihre Effektivität, ihre Effizienz bzw. auf ihr Rationalisierungspotenzial.

Dieser Versuch sollte allerdings nicht dahingehend missverstanden werden, als ob das Jugendgefängnis generell in eine günstige Sozialisationseinrichtung transformiert werden könnte: Es ist und bleibt als ultima ratio des Jugendstrafrechts immer diejenige Sozialisationsinstanz, die wegen der zahlreichen mit dem Freiheitsentzug verbundenen Einschränkungen die bei weitem ungünstigsten Voraussetzungen von allen aufweist. Nur darf uns das, so lange es keine bessere Alternative gibt, nicht davon abhalten, den Jugendstrafvollzug so weit zu verbessern wie irgend möglich und insbesondere den zahlreichen negativen Auswirkungen der Haft entgegen zu wirken.
Das Thema wird eng verstanden, sodass ich mich auf die Rahmenbedingungen innerhalb der Vollzugsanstalt beschränke; und auch diese werden nur insoweit diskutiert, als sie für die Förderung der Gefangenen in ihrer Entwicklung von Bedeutung sind. Der äußerst wichtige Punkt der Entlassungsvorbereitung und -nachsorge, der weit darüber hinausreicht, wird deshalb nicht ausführlich behandelt. Trotzdem sei darauf hingewiesen, dass Entlassungsvorbereitung vom ersten Tag der Haft an eine elementare Aufgabe aller Mitarbeiter und des Gefangenen selbst ist. Was die Integrationsaussichten anbelangt und damit die Verhinderung eines Rückfalls, dürften die ersten Wochen nach der Entlassung aus der Haft sogar entscheidend sein. Dabei wird es darauf ankommen, dass der Gefangene nicht einfach nur an andere Institutionen abgegeben wird, sondern von diesen im wörtlichen wie im übertragenen Sinne des Wortes in der Vollzugsanstalt abgeholt wird (Kerner 2003, 28). Das setzt gemeinsame Vorbereitung der Entlassung mit dem Gefangenen selbst, mit der Familie, der Bewährungshilfe, dem zukünftigen Arbeitgeber und allen anderen Beteiligten voraus. Das in der JVA Adelsheim seit Frühjahr 2006 laufende, vom Europäischen Sozialfonds geförderte Projekt „Integration junger Strafgefangener in Arbeit und Beruf (ISAB)“ stellt hier, ähnlich wie die MABISProjekte in Nordrhein-Westfalen, einen viel versprechenden Ansatz dar.

\section{Vorgaben des Bundesverfassungsgerichts}

Entsprechend dem gegebenen Thema soll selbstverständlich keine auch nur annähernd erschöpfende Besprechung des Grundsatzurteils des Bundesverfassungsgerichts vom 31. Mai 2006 (ZJJ 2006: 193) zur Frage einer gesetzlichen Regelung des Jugendstrafvollzugs geleistet werden. Erforderlich ist allerdings eine kurze Darstellung der Vorgaben des Bundesverfassungsgerichts für die inhaltliche Ausgestaltung eines verfassungskonformen Jugendstrafvollzugs.

Ausgangspunkt der Überlegungen ist die Feststellung des Gerichts, dass es für den Jugendstrafvollzug von Verfassungs wegen gesetzlicher Grundlagen bedarf, „die auf die besonderen Anforderungen des Vollzugs von Strafen an Jugendlichen und ihnen gleichstehenden Heranwachsenden zugeschnitten sind“. Wie bereits in zahlreichen früheren Entscheidungen (BVerfGE 35, 202; 36, 174; 45, 187; 64, 261; 74, 102; 98, 169) festgestellt, muss ein verfassungsgemäßer Vollzug der Freiheitsstrafe und selbstverständlich auch der Jugendstrafe auf das Ziel ausgerichtet sein, dem Inhaftierten ein künftiges straffreies Leben in Freiheit zu ermöglichen. Damit kommt dem Resozialisierungsziel, das für den Jugendstrafvollzug einfachgesetzlich in § 91 Abs. 1 JGG als Erziehungsziel verankert ist, Verfassungsrang $\mathrm{zu}$, was das Gericht in den zitierten Entscheidungen sowohl auf die Menschenwürde in Art. 1 Abs. 1 GG wie auch auf den Sozialstaatsgrundsatz stützt. Außer- dem wird betont, dass „für den Jugendstrafvollzug (...) das Ziel der Befähigung zu einem straffreien Leben in Freiheit besonders hohes Gewicht" hat.

In diesem Zusammenhang weist das Bundesverfassungsgericht auf die besondere Bedeutung des Jugendalters hin, weil Jugendliche in ihrer Persönlichkeit weniger verfestigt sind als Erwachsene und ihre Entwicklungsmöglichkeiten offener sind. Außerdem wirke sich der Freiheitsentzug auf Jugendliche in besonders einschneidender Weise aus. „Indem der Staat in diese Lebensphase durch Entzug der Freiheit eingreift, übernimmt er für die weitere Entwicklung des Betroffenen eine besondere Verantwortung. Dieser gesteigerten Verantwortung kann er nur durch eine Vollzugsgestaltung gerecht werden, die in besonderer Weise auf Förderung - vor allem auf soziales Lernen sowie die Ausbildung von Fähigkeiten und Kenntnissen, die einer künftigen beruflichen Integration dienen - gerichtet ist." ${ }^{2}$ Das Gericht macht sich damit die in den Erziehungswissenschaften seit langem herrschende Auffassung zu eigen, dass sich das Jugendalter vom Erwachsenenalter in wesentlichen Hinsichten unterscheidet und daher von Verfassungs wegen strafrechtlich und auch strafvollzugsrechtlich anders als dieses zu behandeln ist. Sind schon die Ausgangsbedingungen und Folgen strafrechtlicher Zurechnung nach dem JGG bei Jugendlichen und Heranwachsenden anders als bei Erwachsenen geregelt, so hat dies ebenso für den Jugendstrafvollzug und seine rechtlichen Regelungen zu gelten. „Aus alledem ergeben sich spezielle Bedürfnisse, besondere Chancen und Gefahren für die weitere Entwicklung und eine besondere Haftempfindlichkeit, vor allem auch eine spezifische Empfindlichkeit für mögliche schädliche Auswirkungen des Strafvollzugs."

Daraus wird die Konsequenz gezogen, dass Gesetzgebung und praktische Gestaltung des Jugendstrafvollzuges diesen Besonderheiten von Verfassungs wegen Rechnung zu tragen haben. Das Gericht findet sie hauptsächlich in den folgenden wichtigen Bereichen:

Bei allen Vollzugsmaßnahmen, die in Grundrechte eingreifen. Dies ist namentlich der Fall im Hinblick auf Außenkontakte der Gefangenen, auf ihr jugendgemäß besonders großes Bedürfnis für körperliche Betätigung und Bewegung, aber auch im Hinblick auf die Regelung der Sanktionierung von Pflichtverstößen.

In Übereinstimmung mit zahlreichen wissenschaftlichen Erkenntnissen sieht das Gericht weiter besonderen Regelungsbedarf bei der Ausgestaltung des gerichtlichen Rechtsschutzes für die im Jugendstrafvollzug Inhaftierten.

Schließlich verpflichtet das Bundesverfassungsgericht den Gesetzgeber, bei der Entwicklung eines wirksamen Erziehungs- und Resozialisierungskonzepts die genannten Besonderheiten 
des Jugendalters im Unterschied zum Erwachsenenalter zu berücksichtigen. „Der Gesetzgeber muss vorhandene Erkenntnisquellen, zu denen auch das in der Vollzugspraxis verfügbare Erfahrungswissen gehört, ausschöpfen (vgl. BVerfGE 50, 290[334]) und sich am Stand der wissenschaftlichen Erkenntnisse orientieren (vgl. BVerfGE 98, $169[201]) . “$

Insbesondere der letzte Punkt soll nachfolgend im Zentrum stehen, und zwar durchaus im Zusammenhang mit der vom Bundesverfassungsgericht außerdem gemachten Auflage (BVerfG aaO: 197), dass „die gesetzlichen Vorgaben für die Ausgestaltung des Vollzuges (...) auf sorgfältig ermittelten Annahmen und Prognosen über die Wirksamkeit unterschiedlicher Vollzugsgestaltungen und Behandlungsmaßnahmen beruhen" müssen und dass der Gesetzgeber ebenso wie die für den Jugendstrafvollzug zuständigen Behörden verpflichtet ist, aus den mit den bisherigen Vollzugsgestaltungen gemachten Erfahrungen zu lernen“ (vgl. Flügge, Maelicke, Preusker 2001). Einen theoriegeleiteten und evidenzbasierten Jugendstrafvollzug zu entwickeln ist also die der Gesetzgebung und der Vollzugspraxis gestellte Aufgabe.

\section{Ziel des Jugendstrafvollzugs}

ist bereits heute nach der einfachgesetzlichen Regelung in $\S 91$ Abs. 1 JGG, aber ebenso nach der nun präzisierten verfassungsrechtlichen Lage, dass der Gefangene im Jugendstrafvollzug Fähigkeiten erwerben soll, die ein künftiges straffreies Leben in Freiheit und sozialer Verantwortung ermöglichen.

Der langjährige und bis heute nicht endgültig ausgefochtene Streit um den Erziehungsbegriff im Jugendstrafrecht (dazu zuletzt Ostendorf 2006: 320 mit zahlreichen weiteren Nachweisen) und zur Frage, ob das Jugendstrafrecht als solches erzieherisch wirken kann und soll, spielt hierbei nur eine untergeordnete Rolle. Er betrifft in erster Linie das Jugendstrafverfahren und die mit der Verhängung der Jugendstrafe verfolgten Zwecke, kaum jedoch den Jugendstrafvollzug. Vielmehr ging und geht es in erster Linie darum, ob und inwieweit die Verhängung jugendstrafrechtlicher Sanktionen auf Grund „schädlicher Neigungen“ (§ 17 Abs. 2 JGG) sinnvoll sei und inwieweit die Sanktionen des Jugendstrafrechts überhaupt geeignet seien, solche (unterstellten) Erziehungsmängel auszugleichen. Hiervon abgesehen ist wohl unbestritten, dass im Jugendstrafvollzug erzieherische und sozialisationsbezogene Elemente stark gefragt sind und die Zeit der Inhaftierung genutzt werden muss, um schulische und berufliche Aus- und Fortbildung zu betreiben, prosoziale Dispositionen zu unterstützen bzw. entsprechende Ressourcen zu nutzen und etwaige Sozialisationsmängel abzubauen (Scholz 1992: 315 f; Streng 1993: 138,140; Kaiser 1995: 16; Begemann 1991: 280; Walter 2001: 17 ff). Das sehen auch jene Autoren nicht anders, die an- sonsten für die Ablösung des Erziehungsstrafrechts plädieren (P.A. Albrecht 1993: 242 ff, 255 ff; Ostendorf JGG, Grundlagen zu $\S 1$ und 2, Rz. 4,5; Müller 1991: 345 ff). Der Erziehungsgedanke soll als alleiniges Leitprinzip sowohl für die Zielsetzung als auch für die Gestaltungsgrundsätze des Jugendstrafvollzugs dienen (Deutscher Jugendgerichtstag 1995, AK II/6,1996: 264).

Freilich ist der Erziehungsbegriff im gesamten Jugendstrafrecht nicht näher definiert, zudem recht unscharf und für vielerlei Inhalte offen. Deshalb ziehe ich den engeren Terminus „Förderung in der Entwicklung" vor, wie er sich in § 1 SGB VIII findet. Selbstverständlich sind auch damit erzieherische Maßnahmen gemeint; ihre Reichweite und Zeitdauer wird aber von vornherein als begrenzt gedacht. Die damit gewählte Begrifflichkeit ist konkreter, nüchterner und auch weniger ideologisch aufgeladen. Sie bietet den Vorteil, dass "trojanische Pferde" (Gerken/Schumann 1988), "Erziehungslyrik" und überzogene Erwartungen besser außen vor gehalten werden können und die Gefahr verringert wird, dass der Erziehungsgedanke dafür instrumentalisiert werden kann, die Rechte und Handlungsräume der Jugendlichen weiter einzuschränken (Müller-Dietz 1986: 339, 341). Was im Kontext des Jugendstrafvollzuges unter Förderung des Gefangenen in seiner Entwicklung durch erzieherische Angebote und Maßnahmen verstanden wird und wie weit diese reicht, soll deshalb kurz umrissen werden.

\section{Was kann Jugendvollzug leisten?}

Davon ausgehend, dass es in jeder staatlich veranlassten Erziehung im Rechtsstaat allenfalls um einzelne und bestimmte, genau definierte Interventionen geht, ist zu fragen, was der Jugendstrafvollzug nach derzeitigem Wissensstand theoretisch sowie in der regelmäßig sehr begrenzten Zeit an Förderung des Gefangenen in seiner Entwicklung, von der das Bundesverfassungsgericht spricht (aaO: 196), realistischerweise leisten kann. Ich beginne mit dem, was er wohl nicht leisten kann:

Jugendliche und Heranwachsende sind wie alle Menschen keine „Trivialmaschinen“, also nicht im mechanischen Sinne steuerbar, wie manche dies vom Jugendstrafvollzug zu erwarten scheinen. Unter Trivialmaschinen versteht man ja Apparate wie etwa Autos, die per Knopfdruck steuerbar sind: der Input, z.B. der Tritt aufs Gaspedal, führt unmittelbar zu einer Reaktion des Systems, im Beispielsfalle zur Beschleunigung. Völlig anders reagieren dagegen nicht triviale Systeme, wie der Mensch eines darstellt. In diesem Falle stößt der Input eben nicht bloß einen Output an, sondern auch eine schwer vorhersagbare Änderung des inneren Zustandes des Systems. So wird man beispielsweise auf die einfache Frage an einen Gefangenen nach seinen Vorstrafen nicht nur, wenn überhaupt, eine mehr oder weniger zutreffende
Antwort erhalten, sondern in ihm ggf. auch unangenehme Gefühle wecken, womöglich Widerstand aktivieren. Nicht triviale Systeme entziehen sich daher einer einfachen mechanischen Steuerung, sie sind eigen-sinnig, eigen-willig.

Auch mehrfach straffällige junge Menschen, wie wir sie überwiegend im Jugendstrafvollzug antreffen, sind nicht einfach „Defizite der Erziehung". Zwar werden sie nicht selten bestimmte Defizite haben, z.B. im Bereich der Wahrnehmung, der Informationsverarbeitung, der schulischen Bildung und des Sozialverhaltens. Schwerpunktmäßig an solchen Defiziten anzusetzen ist aber aus mindestens zwei Gründen problematisch. Zum einen wäre ein solcher Ansatz überhaupt nur sinnvoll, wenn Möglichkeiten zur Beseitigung konkret vorhanden sind. Das ist aber häufig gerade nicht der Fall: Jugendstrafvollzug kann allgemeine Probleme für die Entwicklung Jugendlicher wie Schulmisere, Lehrstellenmangel, Arbeitslosigkeit nicht beeinflussen, Chancenlosigkeit nicht hinweg reden. Pädagogik, wie sie im Jugendstrafvollzug gefordert ist, kann Struktur- und Sozialpolitik nicht ersetzen. Zum anderen aber erscheint ein defizitorientierter Ansatz wegen der Gefahr frühzeitiger Etikettierung nicht sinnvoll und sogar gefährlich. In den meisten Fällen wird es vielmehr nahe liegen zu versuchen, an den Kompetenzen, Begabungen und Stärken eines jungen Menschen anzusetzen, die in seinem früheren und jetzigen Leben aufzuspüren wären, ohne seine Schwächen und Defizite deshalb zu übersehen.

Dem Jugendstrafvollzug nicht möglich und aus verfassungsrechtlicher Sicht auch nicht gestattet wäre es schließlich, eine totale Persönlichkeitsumwandlung anzustreben (Ostendorf JGG §§ 91-92 Rz. 11, Pollähne 2005: 79, [FN 32]). Vielmehr geht es unter der notwendigen Einschränkung „begrenzter Zuständigkeit“" (Oelkers 2000, 257) in jeder Erziehung im pluralistischen und demokratischen Rechtsstaat allenfalls um einzelne Maßnahmen und bestimmte, genau definierte Interventionen. Dabei steht im Vordergrund die Förderung eines Gefangenen in seiner Entwicklung, wie das $\S 1$ Abs. 1 SGB VIII als Recht aller jungen Menschen statuiert. In zweiter Linie ist das Problem strafbarer Handlungen, der Schädigung anderer oder, ganz abstrakt formuliert, das Zuwiderhandeln gegen die demokratisch legitimierte Rechtsordnung ins Auge zu fassen. Nicht dagegen geht es um die Änderung der Gesamtpersönlichkeit, des gesamten Lebensentwurfes und sämtlicher Einstellungen und Verhaltensweisen des Gefangenen.

Obgleich das Mindestmaß der Jugendstrafe immerhin 6 Monate beträgt, wurden die allermeisten im Vollzug befindlichen Jugendlichen und Heranwachsenden doch zu eher kurzen Strafen verurteilt, in Baden-Württemberg im Jahre 2004 im Durchschnitt zu 1 Jahr und 9 Monaten. Und sie werden zu einem vergleichsweise hohen Prozentsatz gem. § 88 JGG vorzeitig zur Bewäh- 


\begin{tabular}{ll}
\hline Allgemeine Rückfallquote (Wiederkehrerquote) nach 4-5 Jahren: & $56 \%$ \\
\hline Berufsausbildung im Jugendstrafvollzug mit Abschluss: & $21 \%$ \\
Untergebracht überwiegend im gelockerten Vollzug: & $37 \%$ \\
Nach Entlassung in Arbeit: & $46 \%$ \\
Schulabschluss im Jugendstrafvollzug: & $51 \%$ \\
Berufsausbildung im Jugendstrafvollzug ohne Abschluss: & $51 \%$ \\
Urlaub oder Ausgang erhalten: & $52 \%$ \\
Entlassung zur Bewährung: & $53 \%$ \\
\hline Entlassung zum Strafende: & $62 \%$ \\
Überwiegend im geschlossenen Vollzug untergebracht: & $63 \%$ \\
Weder an Schule noch an Berufsausbildung teilgenommen: & $64 \%$ \\
Arbeitslos nach Entlassung: & $64 \%$
\end{tabular}

rung oder gemäß $\S 35$ BtMG in Therapie entlassen $^{3}$. In Bezug auf die tatsächliche Verweildauer bedeutet das für Baden-Württemberg (2005), dass die Gefangenen sich im Durchschnitt ca. 11 Monate im Jugendstrafvollzug befinden. Allerdings ist die Verteilung schief: Die Hälfte bleibt sogar weniger als 10 Monate. Dass in solch kurzer Zeit in der großen Mehrzahl der Fälle eine „nachholende Gesamterziehung" gar nicht möglich ist, sondern allenfalls eine begrenzte Förderung, liegt auf der Hand.

Wie und womit könnte man demgegenüber nach bisherigen wissenschaftlichen Befunden und praktischen Erfahrungen die Entwicklung der Jugendlichen und Heranwachsenden fördern? Oder anders gefragt, wie kann der Gefangene im Jugendstrafvollzug bestmöglich darin unterstützt werden, die ihm altersspezifisch gestellten Entwicklungsaufgaben produktiv zu bewältigen? Eine zwar sehr allgemeine, aber treffende Antwort hierauf hat Kaiser $(1995,16)$ schon vor Jahren gegeben, als er bemerkte, dass alles, was außerhalb des Jugendstrafvollzuges für die gedeihliche Entwicklung junger Menschen für erforderlich gehalten wird, gerade für Straffällige nicht entbehrlich sein kann - und das sind natürlich und zu allererst schulische und berufliche Bildung, körperliche Ertüchtigung und soziales Lernen.

Über diese allgemeine Antwort hinaus kann uns eine differenzierte Rückfallforschung Hinweise geben, welche Vollzugsgestaltungen geeignet erscheinen, dem Ziel der Befähigung zu einem straffreien Leben näher zu kommen - und welche eher nicht. Schlagwortartig, nun allerdings auf Vollzugsmaßnahmen und Organisationsstruktur bezogen, kann man es auch in die Fragestellungen des amerikanischen Sherman-Reports kleiden, die lauten: „What works? What doesn't? What's promising"?

Die statistisch bedeutsamsten Risikofaktoren für Straffälligkeit im Allgemeinen und Rückfall nach Strafvollzug im Besonderen sind bekanntlich männliches Geschlecht und jugendliches Alter. Bei einem Entlassungsalter von etwa 20 Jahren für die aus dem Jugendvollzug entlassenen Gefangenen treffen diese beiden wirkmächtigsten Faktoren zusammen, so dass schon deshalb hohe Rückfallquoten nach Jugendstrafvollzug von vornherein zu erwarten sind. Trotzdem ist es natürlich nicht gleichgültig, was im Jugendstrafvollzug mit den Insassen geschieht oder unterlassen wird. Es empfiehlt sich daher ein Blick auf die unterschiedlichen Rückfallraten, die sich ergeben, wenn man nach der Gestaltung des Jugendstrafvollzugs im Einzelfall differenziert. Solcherart differenzierte Daten enthalten die meisten Rückfallstatistiken jedoch nicht. Sie messen oft nur, ob in einem bestimmten Zeitraum nach Entlassung aus dem Strafvollzug erneut strafbares Verhalten aufgetreten ist, fragen aber nicht, wie lange Zeit ein Verurteilter im Jugendstrafvollzug verbracht hat, in was für einer Anstalt das gewesen ist, was mit ihm dort geschehen ist, welche Beziehungen er während dessen eingegangen ist, welche Erfolge oder Misserfolge er gehabt hat, ob beispielsweise eine schulische oder berufliche Qualifizierung möglich war usw.

Ich beziehe mich deshalb auf eine Arbeit von Dolde \& Grübl $(1996,221)$, die wenigstens einige wichtige Unterdifferenzierungen der auf $56 \%$ für die gesamte Entlassenenkohorte berechneten Rückfallrate (i.S. erneuter Inhaftierung) enthält (siehe Tabelle).

Es zeigt sich hier, dass die Gestaltung des Jugendstrafvollzugs im Einzelfall offensichtlich Einfluss auf die Wiederkehrerquote haben kann. Besonders beeindruckend ist, dass diese bei denjenigen, die im Vollzug einen Beruf bis zum Abschluss erlernt hatten, mit $21 \%$ sehr niedrig ist, aber auch dass diejenigen, die überwiegend im gelockerten Vollzug untergebracht werden konnten, mit $37 \%$ überdurchschnittlich gut abschnitten - ein Ergebnis, zu dem auch zahlreiche andere Studien ge- kommen sind (vgl. unten These 6). Wenn es gelang, den Jugendlichen unmittelbar in Arbeit zu entlassen oder wenn ein Schulabschluss erreicht wurde, lag die Quote immer noch deutlich unter dem Durchschnitt.

In ihrem zweiten Abschnitt mit überdurchschnittlich häufigem Rückfall zeigt die Tabelle, dass diejenigen, die nicht vorzeitig zur Bewährung, sondern zum Strafende entlassen wurden, die überwiegend im geschlossenen Vollzug untergebracht oder weder an Schule noch an Berufsausbildung teilgenommen hatten, deutlich ungünstiger als der Durchschnitt abschneiden. Der hohe Prozentabstand zwischen denjenigen, bei denen es gelang, sie in Arbeit zu entlassen (46\%) und denjenigen, bei denen dies nicht gelungen ist (64\%), weist auf die Bedeutung dieses Faktors hin.

Zwar ist zuzugeben, dass die den unterschiedlichen Vollzugsgestaltungen korrespondierenden Rückfallquoten in erheblichem Umfang auch vorselektive Faktoren widerspiegeln, beispielsweise in dem Sinne, dass im gelockerten Vollzug eben eher die günstig prognostizierten Gefangenen untergebracht werden oder dass eine anspruchsvolle Berufsausbildung mit Abschluss eher die Intelligenteren und Durchhaltefähigeren schaffen. In ihrem gesamten Umfang können die gefundenen Unterschiede damit jedoch sicher nicht erklärt werden. Das zeigt sich z. B. an dem hohen Prozentsatz derjenigen, die nach Entlassung in die Arbeitslosigkeit wieder rückfällig werden. Denn schon seit vielen Jahren ist der Einfluss sowohl der Institution Jugendstrafvollzug wie auch des einzelnen Entlassenen darauf, ob es gelingt, ihn in Arbeit zu vermitteln, eher gering. Es sprechen somit neben dem gesunden Menschenverstand auch die Befunde aus Rückfalluntersuchungen dafür, dass unterschiedliche Vollzugsgestaltung zu unterschiedlich hohen Rückfallquoten beitragen kann. Es empfiehlt sich deshalb, diejenigen Vollzugsmaßnahmen zu forcieren, die mit einer verringerten Rückfallquote verbunden sind - und umgekehrt diejenigen nach Möglichkeit zu vermeiden, die offenbar zu einer erhöhten Rückfallquote führen.

Auch wenn nach alledem im Folgenden bescheiden von Förderung des Jugendlichen oder Heranwachsenden im Jugendstrafvollzug durch erzieherische Angebote die Rede ist und das überaus anspruchsvolle Postulat der „nachholenden Gesamterziehung", wie es der BGH mehrfach formuliert hat (grundlegend BGHSt 11, 169), vermieden wird, sollten wenigstens die wichtigsten der dahinter stehenden pädagogischen Grundannahmen dargelegt werden:

- Ich gehe davon aus, dass nicht nur straffällige Jugendliche, sondern alle Menschen der Erziehung, Zivilisierung und Kultivierung bedürfen, dass Zivilisation, humaner Umgang und ein halbwegs gelingendes Miteinander nichts Selbstverständliches sind, sondern ständiger Erarbeitung, Bewusstmachung, Unterstützung 
und Verteidigung bedürfen. Erziehung oder, bescheidener, Förderung in der Entwicklung ist also notwendig. Im Übrigen kann man, wie immer man es auch versuchen mag, als Vollzugsbeamter, Lehrer, Ausbildungsmeister, nicht nicht erziehen: Man erzieht immer, selbst wenn man es bewusst nicht wollte (näher Walter 2001: 18). Wenn aber Erziehung im Jugendstrafvollzug in jedem Falle stattfindet, dann hoffentlich nicht unbewusst, konzeptionslos und unkontrolliert, sondern besser reflektiert, methodisch abgesichert, an überprüfbaren Kriterien orientiert, hauptsächlich aber rechtlich kontrolliert. - Ganz bewusst spreche ich auch von erzieherischen Angeboten. Auch wenn Jugendgefängnisse nach wie vor totale Institutionen sind (zum Gefängnis als totale Institution vergleiche auch die neuere Darstellung von Goffmans Theorie bei Pecher, 2004: 310), gilt für die Erziehung dort, wie in jeder Erziehungseinrichtung, das Sprichwort: „Man kann den Hund nicht zum Jagen tragen“. Zwangsweise Gelerntes, soweit es das überhaupt gibt, hat bestenfalls kurzzeitig Bestand. Im Gedächtnis dominant abgespeichert wird dann nämlich weniger der erwünschte Inhalt als die angewandte Methode: Der Zwang. Das bedeutet freilich nicht, dass man es an Motivation und Stimulation fehlen lassen darf. Im Gegenteil: Erziehung ohne Lernanreize im Sinne positiver Sanktionen wird keinen Erfolg haben. Erst recht wird scheitern, wer glaubt, das Erziehungsziel - ein Leben ohne Straftaten in sozialer Verantwortung - hauptsächlich mit Druck zu erreichen.

- Des Weiteren haben wir es nach meiner Erfahrung im Jugendstrafvollzug mit durchaus „normalen“ jungen Männern zu tun ${ }^{4}$, die ganz überwiegend nicht als Menschen mit einer abweichenden, abnormen Persönlichkeitsstruktur angesehen werden können. Daran ändert es auch nichts, wenn bei ihnen häufig eine Reihe von sozialisatorischen Belastungen und Defiziten zu verzeichnen sind, andererseits aber auch zahlreiche entwicklungsfähige Stärken und Begabungen. Zweitens gehe ich mit Fend (2000: 207) davon aus, dass Jugend prinzipiell die Möglichkeit hat, sich eigenständig zu entfalten: „Sie kann eine eigene Stimme erwerben und sie kann Eigenintentionalität entwickeln. Dies gilt prinzipiell, faktisch dürfen die Abhängigkeiten von biologischen Vorgaben und gesellschaftlichen Einflüssen nicht unterschätzt werden". Außerdem ist zu berücksichtigen, dass nicht alle jungen Menschen die sich ihnen im Jugendalter stellenden Entwicklungsaufgaben gleich erfolgreich bzw. im gleichen Tempo bewältigen.

- Ich sehe die im Jugendvollzug inhaftierten jungen Menschen als grundsätzlich - freilich nicht im selben Umfang wie Vollerwachsene - für ihr Verhalten verantwortlich an. Sie haben Fehler und Straftaten begangen, sind aber auch prinzipiell lernfähig und lernwillig. Dabei werden die inzwischen gut gesicherten kriminologischen Befunde nicht übersehen, die davon ausgehen, dass leichte und mittelschwere Jugendkrimina- lität bei männlichen Jugendlichen als normal (im statistischen und entwicklungspsychologischen Sinne), als ubiquitär, also weit verbreitet und als episodenhaft anzusehen ist (näher 1. periodischer Sicherheitsbericht [PSB], 2001: 475 ff). Dieses Wissen kann vor vorschneller Etikettierung oder Diskriminierung schützen, ebenso vor Skepsis und Pessimismus oder gar Aufgabe der straffälligen Jugendlichen als „unverbesserlich".

Die Fragen, auf die sich die nachfolgenden Thesen beziehen, sind sowohl theoretischer wie praktischer Natur: Auf welcher theoretischen und organisatorischen Grundlage wird nach welchen Methoden gearbeitet, welche personellen und sächlichen Ressourcen werden zur optimalen Zielerreichung benötigt? Wie vom Bundesverfassungsgericht vorgegeben, sind sowohl wissenschaftliche Befunde als auch praktische Erfahrungen berücksichtigt. Dabei stütze ich mich auf zahlreiche theoretische Arbeiten, empirische Untersuchungen und Praxisberichte.

Welches sind nun also, losgelöst von dem augenblicklichen gesamtgesellschaftlichen Kontext, jedoch auf der Basis wissenschaftlicher Theorie, empirischer Befunde und vielfältiger praktischer Erfahrungen, mithin theoriegeleitet und evidenzbasiert, jene Bedingungen einer Förderung junger Menschen im Jugendstrafvollzug, die heute als optimal bezeichnet werden können? Ich stelle 32 Thesen zur Diskussion:

\section{Die Ebene des Inhaftierten}

1. Die Institution gewährt intern genügend Raum und Freiheit, damit sich die Gefangenen aktiv mit einer Lernprozesse stimulierenden Umwelt auseinandersetzen können.

2. Jeder Gefangene hat eine für ihn persönlich zuständige und verantwortliche Bezugsperson aus dem Kreis der hauptamtlichen Mitarbeiter. Diese bemüht sich darum, dass seine Begabungen und Stärken, seine Entwicklungspotentiale und Ressourcen erkannt und unterstützt werden (Empowerment) und dass etwaige Defizite ausgeglichen werden (Kompensation).

3. Dem Bedürfnis der Gefangenen nach Kommunikation untereinander und mit dem Personal wird so weit wie möglich entgegengekommen. Kontakte zu Angehörigen und zur Außenwelt werden gefördert.

4. Die Lebensbedingungen in der Anstalt entsprechen gesunden, anregenden und geordneten Lebensverhältnissen außerhalb.

5. Anstaltsarchitektur und Anstaltseinrichtungen wirken nicht bedrohlich, sondern sind qualitätsvoll, freundlich und anregend. Das gilt besonders für die Hafträume und die Gemeinschaftsräume. Jedem Gefangenen steht ein eigener Haftraum zu.

6. Vollzugslockerungen und Urlaub sind für jeden Gefangenen zum frühesten verantwortbaren Zeit- punkt vorgesehen. Jeder, bei dem nicht Flucht- oder Missbrauchsgefahr vorliegt, wird in den offenen Vollzug eingewiesen.

\section{Die Ebene des Personals}

7. Die Anstalten sind in allen Bereichen mit der erforderlichen Anzahl qualifizierter Mitarbeiter ausgestattet. Das zahlenmäßige Verhältnis der Mitarbeiter der einzelnen Dienste zur Anzahl der Gefangenen ist durch Schlüsselzahlen festgelegt.

8. Alle erzieherisch tätigen Mitarbeiter (also auch die des Allgemeinen Vollzugsdienstes) verfügen über eine pädagogische (Zusatz-) Ausbildung, mindestens den Abschluss einer Fachhochschule oder Fachschule. Sie arbeiten im Team auf fachlicher Grundlage zusammen.

9. Die Mitarbeiter sind an kriminalpädagogischer Arbeit auf wissenschaftlicher Grundlage interessiert und arbeiten engagiert. Sie beachten neue Entwicklungen im Fachbereich und bekommen Gelegenheit, sich fachlich fortzubilden. Sie erhalten Supervision.

10. Die Mitarbeiter sind sich stets möglicher schädlicher Wirkungen des institutionalisierten Freiheitsentzuges bewusst und versuchen, diese zu vermeiden bzw. ihnen kompensatorisch entgegen zu wirken.

11. Die Mitarbeiter gehen vorbildlich und sparsam mit den zur Verfügung stehenden Ressourcen um.

\section{Die Beziehung zwischen Personal und Ge-} fangenen

12. Mitarbeiter im Jugendstrafvollzug haben eine positive Einstellung sowohl zu den Gefangenen wie auch zu der ihnen gestellten Erziehungsaufgabe.

13. Die Mitarbeiter begegnen den Gefangenen offen und freundlich zugewandt, immer aber korrekt. Sie haben in ihrer Ausbildung kommunikative und interkulturelle Kompetenzen erlernt und entwickelt. Sie suchen daher von sich aus (proaktiv) einen Zugang zu den Gefangenen. Sie sind in der Lage, sich in die Lebens- und Entwicklungssituation junger Menschen hineinzuversetzen und die Welt auch mit ihren Augen zu sehen (Perspektivenübernahme).

14. Die Mitarbeiter richten den Blick in erster Linie auf die (ggf. aufzuspürenden) Begabungen und Fähigkeiten der Gefangenen. Sie vertrauen insoweit auf die Entwicklungsfähigkeit junger Menschen und unterlassen negative Etikettierungen.

15. Gleichwohl berücksichtigen die Mitarbeiter, dass viele Jugendstrafgefangene im Bereich des schulischen, beruflichen und sozialen Lernens auf eine Negativkarriere zurückblicken und durch eine Vielzahl von Misserfolgserlebnissen entmutigt und in ihrer Entwicklung beeinträchtigt sind. Die Mitarbeiter ermutigen deshalb die Gefangenen in ihren Anstrengungen und Aktivitäten und versuchen, ihnen Erfolgserlebnisse zu ermöglichen.

16. Das Ermöglichen von Lernen und von Entwicklungsfortschritten geht vor arbeitssparenden An- 
staltsroutinen.

17. Die Mitarbeiter kennen die Bedeutung der Gleichaltrigengruppe und stellen sie in den Mittelpunkt der Erziehungsarbeit. Sie ermöglichen und unterstützen, dass die Gefangenen Verantwortung für sich und andere übernehmen.

18. Gegenüber medial vermittelten Weltsichten sind die Mitarbeiter kritikfähig; sie beachten die Risiken übermäßigen und unreflektierten Medienkonsums. Ihnen ist daran gelegen, den Gefangenen eigene wichtige (Primär-) Erfahrungen mit ihrer Umwelt zu ermöglichen.

19. Soweit irgend möglich, wird versucht, Eltern und andere Angehörige der Gefangenen in die Arbeit einzubeziehen.

20. Es besteht ein elaboriertes System positiver Verstärkung erwünschter, prosozialer Verhaltensweisen. Negative (Straf-) Sanktionen werden dagegen allenfalls ausnahmsweise und nur dann angewandt, wenn sie die Beziehung zu den Bezugspersonen nicht zerstören und eine aufbauende Komponente haben.

21. Sozialem Lernen und insbesondere gewaltlosem Umgang mit Konflikten wird in der Erziehungsarbeit ein hoher Rang eingeräumt.

22. Die Entwicklung der Jugendstrafgefangenen wird dokumentiert unter besonderer Berücksichtigung ihrer Lernfortschritte. Die getroffenen Beobachtungen und Einschätzungen werden dem Gefangenen fortlaufend zurückgemeldet.

\section{Die Lernumwelt und Anstaltsstruktur}

23. Die Bereitstellung von vielfältigen Lernangeboten und Lernhilfen hat absoluten Vorrang.

24. Die sächliche Ausstattung der Anstalten ist zweckentsprechend, qualitätsvoll und auf neuem technischen Stand (keine Mangelwirtschaft).

25. Die Größe der Wohngruppen ist an den langjährigen Erfahrungen in der Gruppenarbeit orientiert (ca. 8 Mitglieder).

26. Die Strukturen in der Anstalt laden Gefangene und Mitarbeiter zur Partizipation ein und können von ihnen als fair und gerecht wahrgenommen werden. Das Anstaltsklima muss positiv gestaltet werden.

27. Die Struktur und Organisation der Anstalt wird ständig auf ihre "Jugendverträglichkeit" sowie ihre Eignung für eine effiziente Zielerreichung bestmögliche Förderung der Gefangenen in ihrer Entwicklung - überprüft. Eine fortlaufende Kontrolle der Zielerreichung anhand überprüfbarer Kriterien wird implementiert.

28. Bezüglich der schulischen und beruflichen Fortbildung besteht ein professionelles Beratungssystem, das Gefangenen und Mitarbeitern jederzeit zur Verfügung steht.
29. Jede Jugendstrafanstalt hat eine eigene, selbständige Schule, die als Ganztagesschule geführt wird.

30. Die schulischen und beruflichen Ausbildungseinrichtungen sowie die Sportanlagen des Jugendvollzugs stehen externen Programmteilnehmern grundsätzlich offen.

31. Die Justizvollzugsanstalt pflegt enge Zusammenarbeit mit Institutionen wie Schulen, Ausbildungszentren, Therapieeinrichtungen, Heimen und Forschungseinrichtungen, die im Bereich der Erziehung, Bildung und Jugendhilfe tätig sind.

32. Die Aufsichtsbehörden beschränken sich auf die Fach- und Rechtsaufsicht sowie die Globalsteuerung. Sie widerstehen der Versuchung, im Einzelfall mitzuerziehen.

Dr. Joachim Walter ist Leiter der Jugendstrafanstalt Adelsheim, Baden-Württemberg, und Mitherausgeber dieser Zeitschrift

\section{Literatur:}

Albrecht, Peter-Alexis: Jugendstrafrecht, 2. Auflage München 1993.

Begemann, Helmut: Zur Legitimationskrise der Jugendstrafe. DVJJ-Journal 1991,

Bundesministerium des Innern (Hrsg.): Erster Periodischer Sicherheitsbericht (PSB), Berlin 2001.

Callies, Rolf-Peter/Müller-Dietz, Heinz: Strafvollzugsgesetz. Kommentar. 10. Aufl. München 2005

Dolde, Gabriele/Grübl, Günter: Jugendstrafvollzug in Baden-Württemberg. Untersuchungen zur Biographie, zum Vollzugsverlauf und zur Rückfälligkeit von ehemaligen Jugendstrafgefangenen, S. 221. In: Kerner, Hans-Jürgen/Dolde, Gabriele/Mey, Hans-Georg (Hrsg.): Jugendstrafvollzug und Bewährung. Analysen zum Vollzugsverlauf und zur Rückfallentwicklung. Bonn 1996, S. 221.

Deutsche Vereinigung für Jugendgerichte und Jugendgerichtshilfen e.V. (DVJJ):

Eckpunktepapier: Anforderungen an ein zukünftiges Jugendstrafvollzugsgesetz. ZJJ 2004, S. 209

Flügge, Christoph/Maelicke, Bernd/ Preusker, Harald (Hrsg.): Das Gefängnis als lernende Organisation, Baden-Baden 2001

Gerken, J./Schumann, K. F.: Ein trojanisches Pferd im Rechtsstaat. Der Erziehungsgedanke in der Jugendgerichtspraxis. Pfaffenweiler 1988.

Kaiser, Günter: Ist der Erziehungsgedanke im Jugendstrafrecht wirklich veraltet? In: Busch, Max/MüllerDietz, Heinz/Wetzstein, Hans (Hrsg.): Zwischen Erziehung und Strafe. Zur Praxis der Jugendstrafrechtspflege und ihrer wissenschaftlichen Begründung. Festschrift für Karl Härringer zum 80. Geburtstag. Pfaffenweiler 1995, S. 9.
Kerner, Hans Jürgen: Der Übergang vom Strafvollzug in die Gesellschaft: Ein klassisches Strukturproblem für die Reintegration von Strafgefangenen. In: Bremer Institut für Kriminalpolitik (Hrsg.): Quo Vadis III, 1. Auflage Bremen 2003, S. 27.

Müller, Siegfried: Erziehen - Helfen - Strafen. DVJJ-Journal 1991, S. 345.

Müller-Dietz, Heinz: 10 Jahre Strafvollzugsgesetz. BewHi 1986, S. 331.

Ostendorf, Heribert: Jugendgerichtsgesetz. Kommentar. 5. Aufl. Köln usw. 2000

Ostendorf, Heribert: Gegen die Abschaffung des Jugendstrafrechts oder seiner Essentialia. NStZ 2006, S. 320

Pecher, Willi: Totale Institution in: Pecher, Willi(Herausgeber), Psychologie in Schlüsselbegriffen, Stuttgart 2004, Seite 310

Pollähne, Helmut: Anmerkung zum Beschluss des OLG Hamm vom 01.07.2004,

1 VAs 17/04, ZJJ 2005, S. 77.

Scholz, Christian: Zur Grundstruktur eines neuen Jugenddevianzrechts. DVJJ-Journal 1992, S. 301.

Streng, Franz: Der Erziehungsgedanke im Jugendstrafrecht. ZStW 106 (1994) S. 60.

Walter, Joachim (2001): Erziehung durch Jugendstrafe Erziehung im Jugendvollzug. In: Bremer Institut für Kriminalpolitik (Hrsg.): Alternativsymposium zum Strafvollzug anläßlich des Erscheinens der 4. Auflage des Alternativkommentars zum Strafvollzugsgesetz (AK-StVollzG) (Materialien zur Kriminalpolitik Band 9) Bremen; S. 15 - 27.

Walter, Joachim (2003): Erwartungen der Praxis an ein künftiges Jugendvollzugsgesetz. ZJJ 4/2003, S. 397 403.

\section{Fußnoten}

1 Stark gekürzte Fassung meines in Heft 3/2006 der ZJJ erschienenen Aufsatzes „Bedingungen bestmöglicher Förderung im Jugendstrafvollzug. Ein Diskussionsbeitrag in der Folge des Urteils des Bundesverfassungsgerichts vom 31.05.2006, ZJJ 2006, S. 193“. Wegen näherer Begründung der Thesen und detaillierter Literaturhinweise darf auf diese Veröffentlichung verwiesen werden.

2 BVerfG aaO: 196; Hervorhebung vom Verfasser.

3 Baden-Württemberg 2005: Entlassung zur Bewährung $49,7 \%$, 35 BtMG 13,8 \% Abschiebung ( $\$ 456$ a StPO) $7,7 \%$, Strafende $28,8 \%$. In früheren Jahren höhere Werte für Entlassung zur Bewährung (1991 = 79,8 \%). 4 Über die sehr kleine Zahl inhaftierter jugendlicher und heranwachsender Frauen wäre gesondert zu sprechen; dies soll hier jedoch sowohl wegen der sehr schmalen Datenlage wie auch insbesondere mangelnder persönlicher Erfahrung unterbleiben. 